\title{
Impact of Mobility on Different Routing Approaches in MANETs
}

\author{
Hrudya K.P. \\ Department of Computer Sc. \& \\ Engineering \\ SRMSCET, Bareilly, India
}

\author{
Brajesh Kumar \\ Department of Computer Sc. \& IT \\ MJP Rohilkhand University, Bareilly, \\ India
}

\author{
Prabhakar Gupta, PhD. \\ Department of Computer Sc. \\ \& Engineering \\ SRMSCET, Bareilly, India
}

\begin{abstract}
In mobile ad hoc networks, the movement of the mobile nodes is one of the important characteristics because it can affect the performance of the routing protocol. The mobility pattern directly influences when communication links between nodes are established or broken, which is associated with the network topology. In this paper, the impact of the different mobility models on the performance of different routing approaches has been studied. AODV, DSR, LAR, and OLSR are taken as the candidate protocols for this purpose. The performance of these protocols is evaluated and analyzed for different mobility models like RWP, RPGM, GM and MH through simulation. The performance metrics like packet delivery ratio, average end-to-end delay, normalized routing load, and throughput are evaluated for these protocols using network simulator NS2 under different scenarios.
\end{abstract}

\section{General Terms}

Mobile Ad hoc Networks, Routing protocols,Mobility models

Key Words- Mobile Ad hoc Networks, Mobility Models, AODV, DSR, OLSR, LAR. , RWP, RPGM, MH, GM

\section{INTRODUCTION}

Wireless mobile communication between mobile users is becoming more popular than ever before due to technological advances in hand held computers and wireless data communication devices. Scalability and flexibility are the key features of wireless networking. In ad hoc networks, there is no fixed infrastructure such as base stations or mobile switching centers. Nodes of an ad hoc network are mobile hosts with similar transmission power and computation capabilities. MHs that are within each other's radio range communicate directly via wireless links. Otherwise, they communicate through multihop routing. Routing is a fundamental issue for networks. To thoroughly and systematically study a new MANET routing protocol, it is important to simulate the protocol and evaluate its performance. Among other parameters mobility is an important parameter for MANETs routing protocols evaluation. To investigate how mobility model affects the performance of a routing protocol is the motivation behind this research. For thorough analysis four routing protocols based on different approaches AODV, DSR, LAR, and OLSR are taken. These protocols are evaluated under four different mobility models Random Waypoint (RWP), Reference Point Group Mobility (RPGM), Gauss Markov (GM), and Manhattan Grid (MG).
The remainder of this paper is structured as follows. The section 2 and section 3 describe briefly the four candidate routing protocols and the mobility models respectively. The review of related work is presented in section 4. Experimental results are shown and discussed in section 5 with concluding remarks in section 6 .

\section{ROUTING POROTOCOLS IN MANETs}

The routing protocols in MANET are broadly classified in to proactive and reactive and each of them suites well in different scenarios.The AODV [14] protocol minimizes the number of broadcasts by creating routes based on demand. When any source node wants to send a packet to a destination, it broadcasts a route request (RREQ) packet. The neighboring nodes in turn broadcast the packet to their neighbors and the process continues until the packet reaches the destination. During the process of forwarding the route request, intermediate nodes record the address of the neighbor from which the first copy of the broadcast packet is received. This record is stored in their route tables, which helps for establishing a reverse path. If additional copies of the same RREQ are later received, these packets are discarded. The reply is sent using the reverse path for route maintenance.

DSR [13] requires mobile nodes to maintain route caches or the known routes. The route cache is updated when any new route is known for a particular entry in the route cache. If a source node wants to send a packet, it first consults its route cache to determine whether it already knows about any route to the destination or not. If not, it initiates a route request broadcast. Each intermediate node checks whether it knows about the destination or not. If the intermediate node does not know about the destination, it again forwards the packet and eventually this reaches the destination. A node processes the route request packet only if it has not previously processed the packet and its address is not present in the route record of the packet. A route reply is generated by the destination or by any of the intermediate nodes when it knows about how to reach the destination.

Location-Aided Routing (LAR) described in [15] uses location information to improve performance of routing protocols for MANET. Position information will be used by LAR for restricting the flooding to a certain area called request zone. As a consequence, the number of route request messages is reduced. It makes use of physical location information of destination node to reduce the search space for route discovery. Instead of flooding the whole network with route discovery message, this protocol send messages to a subset of nodes from whom the probability of finding route is very high. 
OLSR [11] protocol inherits the stability of link state algorithm. This protocol performs hop-by-hop routing; that is each node in the network uses its most recent information to route a packet. Hence, even when a node is moving, its packets can be successfully delivered to it, if its speed is such that its movements could at least be followed in its neighborhood. As only multipoint relays of a node can retransmit its broadcast messages, this protocol significantly reduces the number of retransmissions in a flooding or broadcast procedure.

\section{MOBILITY MODELS}

A mobility model should attempt to emulate the movements of real mobile nodes. Mobility models are based on setting out different parameters related to node movement. Basic parameters are the starting location of mobile nodes, their movement direction, velocity range, speed changes over time.

The RPGM model represents the random motion of a group of mobile nodes and their random individual motion within the group. All group members follow a logical group center that determines the group motion behavior. The entity mobility models should be specified to handle the movement of the individual mobile nodes within the group. Purpose of logical group center is to guide group of nodes continuously.

The RWP model assumes that each host is initially placed at a random position within the simulation area. As the simulation progresses, each host pauses at its current location for a determinable period called the pause time. RWP model assumes the possibility of setting cut-of phase, scenario duration, width and height of the area (x,y) minimum and maximum speed ( $V_{\min }$ and $V_{\max }$ ), as well as maximum pause time. It includes pause times between changes in direction and/or speed. Pause time is used to overcome abrupt stopping and starting in the random walk model. Upon expiry of this pause, the node arbitrary selects a new location to move towards and a new speed which is uniformly and randomly selected from the interval ( $V_{\min }$ and $\left.V_{\max }\right)$.

The Gauss Markov model enables different levels of randomness by setting only one parameter. Initially, each mobile node has preset speed and direction parameter values. This model captures the velocity correlation of a mobile node in time and represents random movement without sudden stops and sharp turns. At fixed intervals of time movement occurs by updating the speed and direction of each node. At each iteration, the new parameter values are calculated depending respectively on the current speed and direction and on a random variable.

The Manhattan grid model has originally been developed to emulate the Manhattan street network, i.e. a city section which is only crossed by vertical and horizontal streets. The trajectories of mobile nodes are confined to a grid topology. The MG model can be described by the following parameters: mean speed, minimum speed (with a defined standard deviation for speed), a probability to change speed at position update, and a probability to turn at cross junctions.

\section{REVIEW OF WORK}

Many studies on performance evaluation of mobile ad hoc routing protocols are found in literature. But most of the studies consider Random Waypoint model. Only few of them also considered other models. A survey of the work is given here.
Simulation studies of MANET routing protocols have mostly assumed Random Waypoint (RW) as a reference mobility model [1], [2]. In order to examine many different MANET applications there is a need to provide additional mobility models. Typical examples are modeling a movement in city streets environment, university campuses and movement of groups of nodes, e.g. for specific military purposes. Recently, a performance comparison of DSR and AODV protocols based on Manhattan Grid (MG) model has been published [3].A performance study of DSR and AODV considering probabilistic random walk and boundless simulation area has been presented in [4].A performance evaluation of DSDV and AODV using scenario based mobility models has been presented in [5]. A comparative analysis of DSR and DSDV protocols, considering RW, Group Mobility, Freeway and MG models can be found in [6].

Authors in [7] studied performance of the three widely used MANET routing protocols (DSDV, AODV and DSR) with respect to group (RPGM) and entity (RW, GM and MG) mobility models. Simulation results have indicated that the relative ranking of routing protocols may vary depending on mobility model. The relative ranking also depends on the node speed as the presence of the mobility implies frequent link failures and each routing protocol reacts differently during link failures. AODV performs best with the group model RPGM. With entity models, AODV experiences the highest routing overhead with the increase of node speed, but has acceptable average delays. DSR experiences the lowest routing protocol overhead, on the count of higher average delays, particularly with MG and GM models, at higher node speeds. This protocol performs best with the RW model.

In [8], the three random based mobility models such as Random waypoint, Random walk and Random Directions were implemented. The two different parameter constraints like packet-delivery fraction and end-to-end packet delivery delay are compared with respect to mobility speed, Traffic and Network size. The simulation results shows that the AODV protocols in Random Waypoint mobility model performs better than DSDV, TORA and DSR in Random walk and random Direction mobility model. The performance of routing protocols with respect to group and entity mobility models is presented in [11]. The DSDV, DSR, and AODV are investigated under RPGM, RWP, GM, and MG.

\section{EXPERIMENTAL RESULTS AND ANALYSIS}

The routing protocols are evaluated using Network Simulator-2 (NS-2) in its version 2.34. The network consists of 50 nodes spread over an area of $1000 \mathrm{~m} * 500 \mathrm{~m}$ with a variation in speed from $4 \mathrm{~m} / \mathrm{s}$ to $40 \mathrm{~m} / \mathrm{s}$ and transmission range 250 meters. One more tool Bonn-Motion is used to generate node movements for different mobility models.

Figure 1 summarizes the packet delivery ratio (PDR) under four different mobility models. It can be observed that PDR for all the four protocols is much higher under RPGM as compared to other models. RWP which is used in most simulation studies is significantly behind. LAR and DSR are consistently best and poorest protocols respectively irrespective of the mobility models. OLSR is the most inconsistent, exhibiting different patterns under different mobility models. 


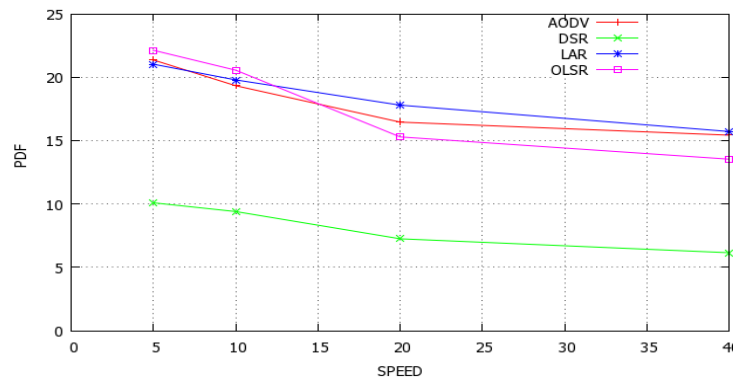

(a) RWP

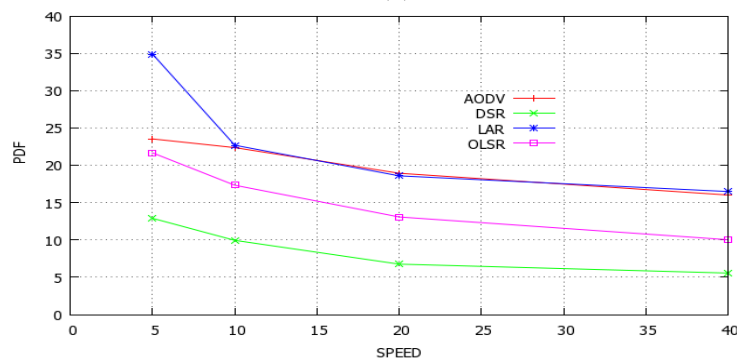

(c) Gauss Markov

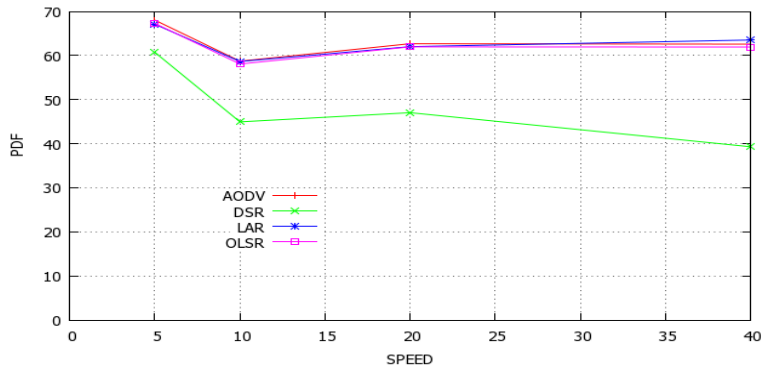

(b) RPGM

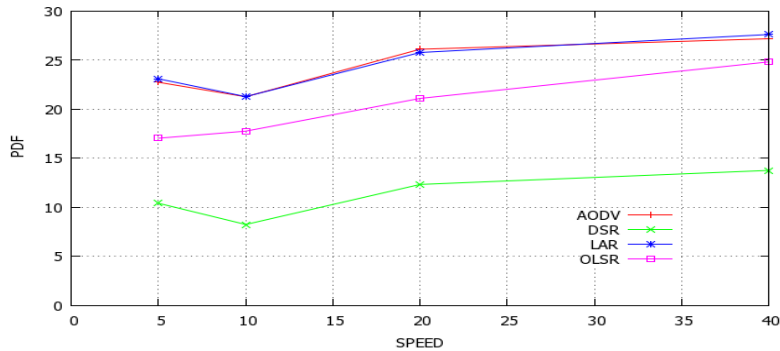

(d) Manhattan Grid

Fig 1: Packet Delivery Ratio

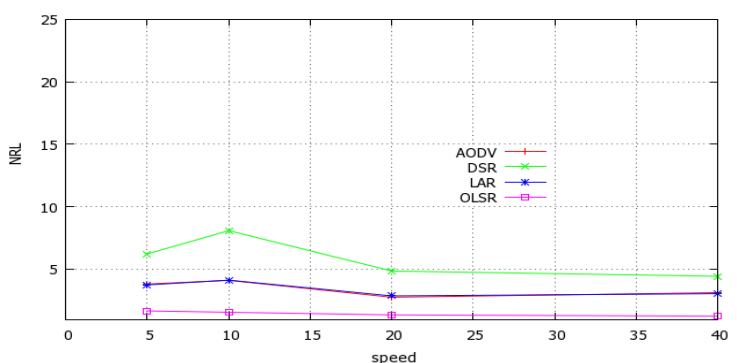

(a) RWP

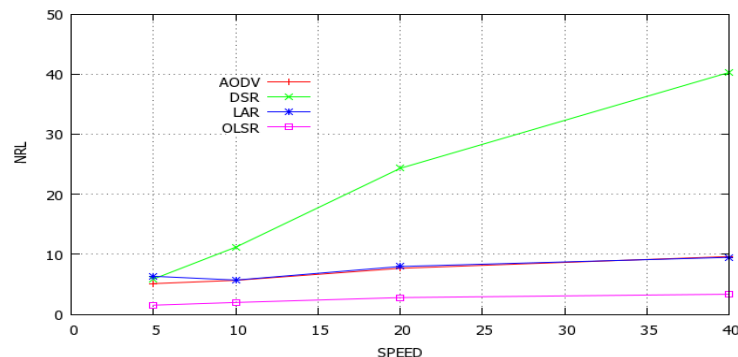

(b) Gauss Markov

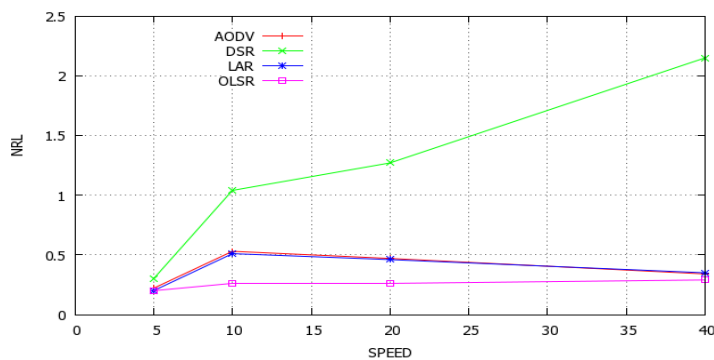

(b) RPGM

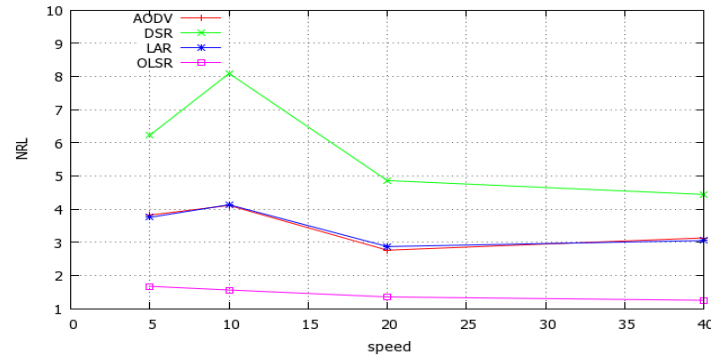

(d) Manhattan Grid

Fig 2: Normalized Routing Load

It can be observed in Figure 2 that mobility model has significant impact on the routing load. Routing load is found to be lowest under RPGM and highest for Gauss Markov model. DSR protocol, which causes the highest overhead is most inconsistent also.
With DSR, overhead is increasing with node speed under RPGM and GM and lower under other mobility models.It is intersesting to observe that OLSR protocol seems to be unaffected by the mobility model in use for this parameter. With OLSR, routing load is almost similiar under all the mobility models. 
Fig 3 shows the measurments of delay at different speeds Again RPGM found to be the best mobility model resulting in lowest delay for all the protocols. The DSR protocol is affected most bythe mobility model. The throughput can be observed in

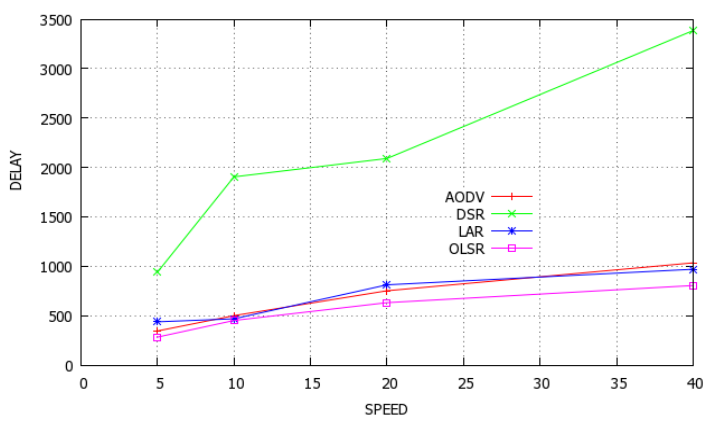

(a) RWP

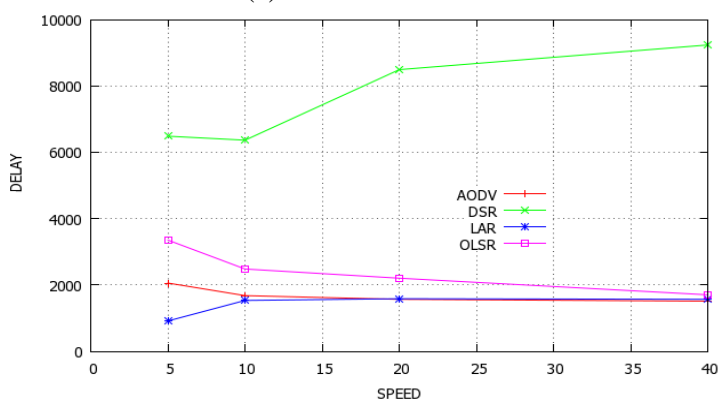

(c) Gauss Markov
Figure 4, which is highest in case of RPGM model.OLSR and DSR are severly affetced by the mobility model for this parameter.

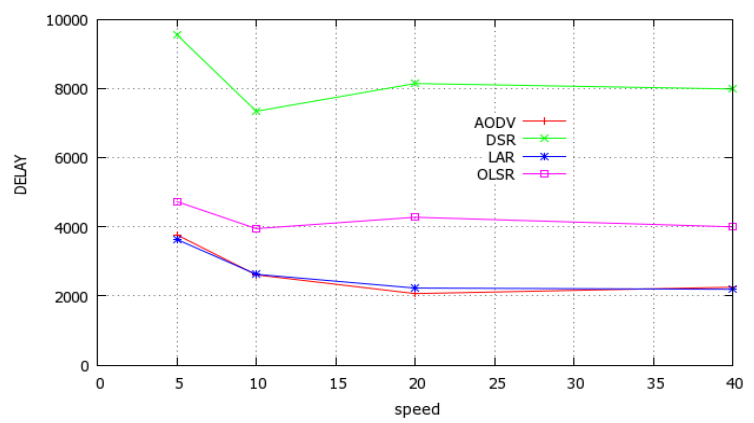

(b) RPGM

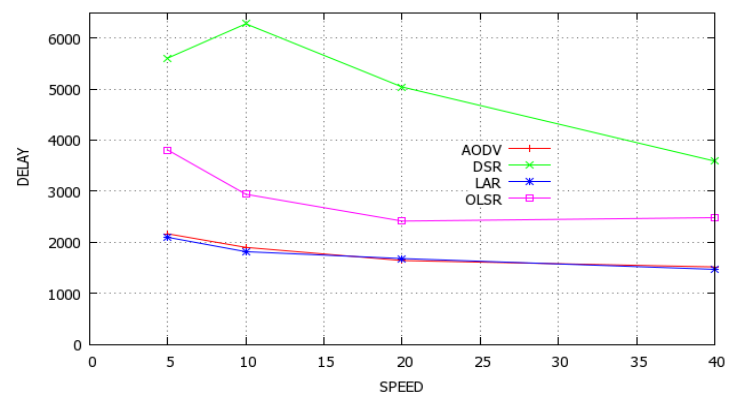

(d) Manhattan Grid

Fig 3: Delay

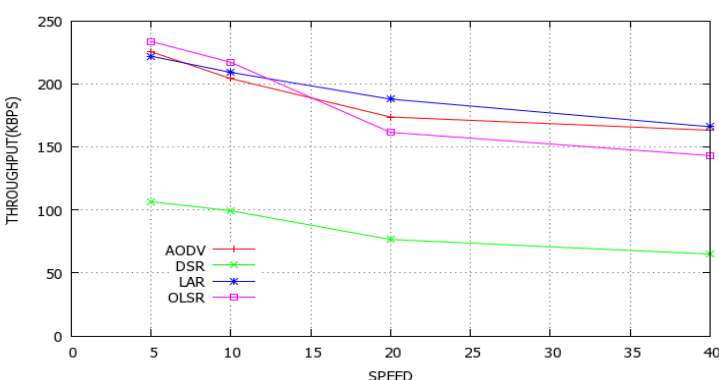

(a) RWP

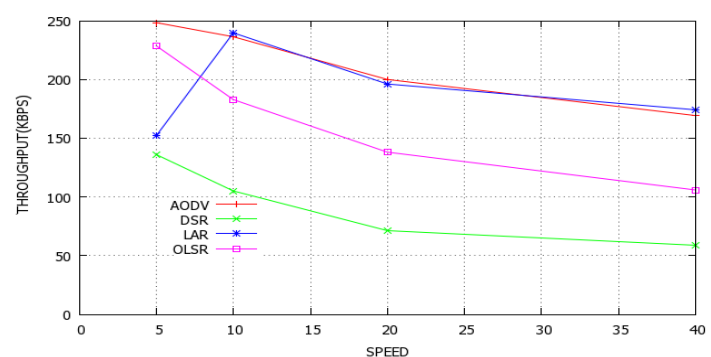

(c) Gauss Markov

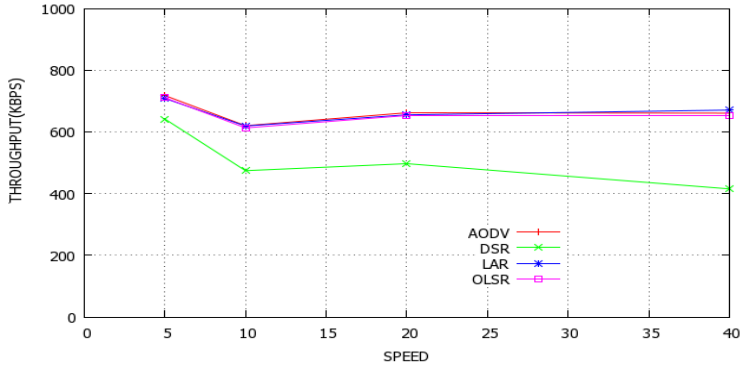

(b) RPGM

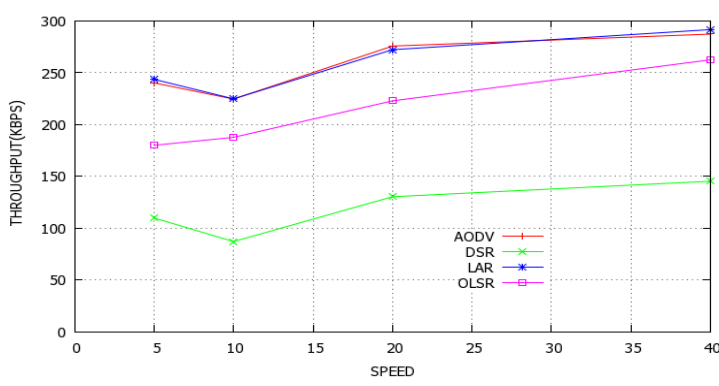

(d) Manhattan Grid

Fig 4: Throughput 


\section{CONCLUSION}

In this paper the impact of the chosen mobility models on the performance of the protocols based on different routing approaches in mobile ad hoc networks was studied and analyzed through simulations. The performance of the protocols was evaluated for the Random Waypoint, RPGM, Gauss Markov, and Manhattan Grid models with varying node speed in terms of packet delivery ratio, average end-to-end delay, normalized routing load, and throughput. Empirically results illustrate that the performance of a routing protocol varies widely across different mobility models. The results shown that chosen mobility model has significant impact on the performance of the routing protocols. There is a considerable difference between the results produced by NS-2 for the different mobility models. If the performance of protocols under a specific mobility model is concerned, it is found that the RPGM model clearly produced the best results. The impact of the mobility models is different on different routing approaches. It is found that DSR and OLSR are most affected by the model chosen

\section{ACKNOWLEDGMENT}

I sincerely and heartedly express my gratitude to Brajesh Kumar, Associate Prof., Department of CSIT, IET, MJP Rohilkhand University, Bareilly who have been very concerned and have aided for all the material essential for the preparation of this paper. I take this opportunity to express my heartful gratitude to Dr.Prabhakar Gupta, Dean Academics, SRMSCET, Bareilly for the motivation and inspiration that triggered me for this work.

\section{REFERENCES}

[1] S. Sesay et al., "Simulation Comparison of Four Wireless Ad Hoc Routing Protocols",Information Technology Journal 3(3), 2004, pp:219-226.

[2] S. Shah et al, "Performance Evaluation of Ad Hoc Routing Protocols Using NS2 Simulation",Conf. of Mobile and Pervasive Computing, 2008

[3] G. Jayakumar, G. Gopinath, "Performance Comparison of MANET Protocol Based on Manhattan Grid Model", Journal of Mobile Communications, vol. 2, no. 1, pp. 1826, 2008 .

[4] M. K. Jeya Kumar, R.S. Rajesh, "A Survey of MANET Routing Protocols in MobilityModels", Int. Journal of Soft Computing 4(3),2009, pp. 136-141

[5] Sham-ul-Arfeen et al, "Performance Evaluation of MANET Routing Protocols Using Scenario Based Mobility Models", Innovative Algorithms and Techniques in Automation, Industrial Electronics and Telecommunications, Springer,2007. pp. 419-424.
[6] B. Divecha et al, "Impact of Node Mobility on MANET Routing Protocols Models", Journal of Digital Information Management, February2007.

[7] Valentina Timcenko, Mirjana Stojanovic, Slavica Bostjancic rakas,"MANET Routing Protocols vs. Mobility Models:Performance Analysis and Comparison", Proceedings of the 9th WSEAS International Conference on Applied Informatics and Communications (AIC '09)

[8] M.K.Jeya Kumar and R.S.Rajesh," Performance Analysis of MANET Routing Protocols in Different Mobility Models", IJCSNS International Journal of Computer Science and Network Security, VOL.9 No.2, February 2009.

[9] T. G. Basavaraju and S. K. Sarkar, "Effect of Random Mobility Models Pattern in Mobile Ad hoc Networks" IJCSNS International Journal of Computer Science and Network Security, VOL.7 No.6, June 2007.

[10] Harminder S. Bindra, Sunil K. Maakar and A. L. Sangal,"Performance Evaluation of Two Reactive Routing Protocols of MANET using Group Mobility Model”, IJCSI International Journal of Computer Science Issues, Vol. 7, Issue 3, No 10, May 2010 ,ISSN (Online): 1694-0784, ISSN (Print): 1694-0814.

[11] Ravinder Ahuja,' Simulation based Performance Evaluation and Comparison of Reactive, Proactive and Hybrid Routing Protocols based on Random Waypoint Mobility Model", International Journal of Computer Applications (0975 - 8887) Volume 7- No.11, October 2010

[12] Gowrishankar.S, SubirKumarSarkar, T.G.Basavaraju," Analysis of AOMDV and OLSR Routing Protocols Under Levy-Walk Mobility Model and Gauss-Markov Mobility Model for Ad Hoc Networks", (IJCSE) International Journal on Computer Science and Engineering Vol. 02, No. 04, 2010, 979-986

[13]C. Adijh, E.Baccelli, and P. Jacquet, "Link state routing in wireless adhoc networks," in MILCOM '03: Military Communications Conference. IEEE Computer Society, 2003, pp. 1274-1279

[14] Zhijiang Chang, Georgi Gaydadjiev, Stamatis Vassiliadis, "Routing Protocols for Mobile Ad-hoc Networks: Current Development and Evaluation", Computer Engineering laboratory, EEMCS, Delft University of Technology Mekelweg 4, 2628 CD Delft, the Netherlands.

[15] Young-Bae Ko and Nitin H. Vaidya,"Location-Aided Routing (LAR) in Mobile Ad hoc Networks." Wireless Networks 6 (2000) 307-321 